\title{
The Research of Routing Algorithm Based on Shortest Path Tree in WSNs
}

\author{
Yuhua Liu, Zhenrong Luo, Ke Xu, Cui Xu \\ Academy of Computer Science, Central China Normal University, Wuhan 430079, China \\ Email: yhliu@mail.ccnu.edu.cn
}

\begin{abstract}
This article poses an algorithm of the hierarchical topology in wireless sensor network based on shortest path tree algorithm to cover the shortage of LAC Routing Algorithm (LAC-T), which based on SPT(Shortest Path Tree). LAC-T algorithm elects cluster head by the remaining energy of nodes and the distance between node and base station. Meanwhile, it uses SPT to communicate among cluster heads in WSNs. The result of stimulation shows that LAC-T algorithm is not only easy to achieve, but could be better balance the energy consumption of each node, extend the lifespan of WSN (Wireless Sensor Network).
\end{abstract}

\section{Keywords- LAC; Energy; Multi-hop; Distance.}

\section{INTRODUCTION}

With the development of communication technology, WSNs which are used to collect, process and transmit information have been widely given more attention. There are a lot of sensor nodes in WSNs. WSNs could collect and process target information through the cooperative work of nodes [1]. The routing technology of network layer is always a hot spot in the research of WSN architecture. WSN is divided into several clusters within hierarchical network routing algorithm. Each cluster includes two types of nodes, cluster heads and ordinary nodes. The cluster heads are in charge of data dissemination of cluster, and exchanges information with other cluster heads. All data are sent into the sink. The typical clumping routing algorithms are LEACH algorithm, and LEACH-C, LAC algorithm which based on LEACH algorithm.

In this article we present the LAC-T algorithm which based on the LAC algorithm. The cluster head election of LAC-T algorithm considers the remaining energy of each node and the distance between node and sink. The communication between nodes is a kind of multi-hop communication which based on SPT (Shortest Path Tree). The result of stimulation shows that LAC-T algorithm can effectively balance the energy consumption of the nodes distribution, extend nodes and network lifespan.

\section{LAC PROTOCOL}

LAC (LEACH and LEACH-C)[3] is a kind of clustering routing algorithm which combines both advantages of LEACH and LEACH-C algorithms. The basic idea of LAC algorithm is to divide target area into several small regions. The election of cluster head is based on random algorithm. The main idea of this random algorithm is that each node produces a random number between 0 and 1 , then compare the random number to a threshold value $T(n)$, if the random number is less than
$T(n)$, the this node is chosen as a cluster head. $T(n)$ is calculated as

$$
T(n)= \begin{cases}\frac{p}{1-p[r \bmod (1 / p)]} & \text { if } n \in G, \\ 0 & \text { otherwise. }\end{cases}
$$

Where $\mathrm{P}$ is the desired percentage to become a cluster head, $r$ is the current round, and $G$ is the set of nodes that have not being selected as a cluster head in the last $1 / p$ rounds.

A table with an ID field is needed in order to identify nodes after the cluster head has been chosen. Cluster heads communicate with the sink through multi-hop method.

As shown in Fig. 1, the LAC protocol divide target region into four homalographic regions, then creates a table to record the region information of all nodes. The ID of each node is calculated based on the distance between node and sink, the ID of the nearest node is one, the ID of nodes is 1 of area C, 2 of area B. Ordinary nodes search the cluster head which has the same ID after receiving data, then send the data to the nearest cluster head, the cluster heads receiving data send the data to another cluster head of which ID number is less one compare to it. Data are integrated in cluster heads of which ID number is 1 , the cluster heads with $\mathrm{ID}=1$ send the processed data to sink.

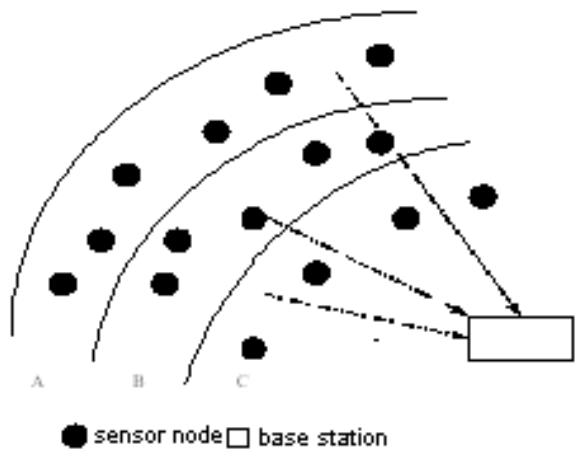

Fig.1. LAC functional scheme.

\section{LAC-T ROUTING MECHANISM}

The improvement of LAC-T algorithm of this article is in that considering the remaining energy of nodes and distance between node and sink while calculating threshold $\mathrm{T}(\mathrm{n})$, for the purpose to balance the energy consumption of nodes, at the communication stage, cluster heads send data through the way of multi-hop 
based on the shortest path tree to sink.

\section{A. Cluster heads threshold selection}

As the randomness of the node distribution, node connectivity is not even. Considering the node connectivity and remaining energy of nodes [4], the modified $\mathrm{T}(\mathrm{n})$ is calculated as:

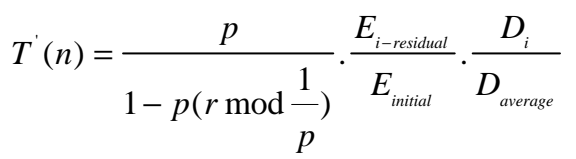

Where $E_{i-\text { residual }}$ is the remaining energy of node; $E_{\text {initial }}$ is the initial energy of node; $D_{i}$ is the distance between node and sink; Daverage is the average distance between node and sink. The modified threshold is better to balance the energy consumption of nodes.

\section{B. Cluster communication based on SPT}

The model of WSN communication is the radio communication model [5]. Within radio communication, the node energy consumption for data transmission is calculated as

$$
E=\left\{\begin{array}{l}
k \cdot E_{\text {elec }}+k \cdot E a m p \cdot d^{2}, d<d_{0} \\
k \cdot E_{\text {elec }}+k \cdot E_{\text {amp }}^{\prime} \cdot d^{4}, d \geq d_{0}
\end{array}\right.
$$

Where $\mathcal{E}_{a m p}$ and $\mathcal{E}_{a m p}^{\prime}$ are energy consumption of node in amplifying circuit to send one bit; $d_{0}$ is the threshold distance. If the communication distance of nodes is less than $d_{0}$, the nodes energy consumption use multipath attenuation model to calculate, so the farther of the distance, the larger of energy consumption. At data transmission stage, using multiple hop transmission among clusters will greatly reduce the energy consumption of cluster head node.

Communication mechanism among clusters based on SPT is described as

At the initial stage, calculate the distance between node and sink according to the strength of signal [6];

(2) After the cluster heads are selected, the cluster heads advertise to all sensor nodes in the network that they are the new cluster heads. Once the sensor nodes receive the advertisement, then they calculate the distance between the cluster head and themselves, then add the distance into the set of cluster heads distance $H$, $H=\left\{d_{i-j} \mid i, j\right.$ are cluster heads $\}$.

(3) Find the shortest path from cluster head i to sink with Prim algorithm. First, select the shortest distance in $\mathrm{H}$, then select the second shortest distance, by this way, the algorithm has calculated i-1 paths before the iteration [7];

(4) Each cluster head calculates its shortest distance to sink with Prim algorithm then sends data to sink along the optimal path.

\section{THE SIMULATION AND ANALYSIS}

There are three types of evaluation criterion to evaluate the wireless sensor networks system. They are FND, HND and LND. FND (First Node Dies) refers to the death time of First Node in WSNs. The performance of the network will significantly decrease after the first node die. Therefore, it's essential to record the death time of the first node. The same reason to record the time of HND(Half Nodes die) and LND (Last Node Dies).

Supposed a network with 100 nodes, the position of each node is randomly disposed in a two-dimensional space. The position of sink is $(x=50, y=75)$, each node is equipped with the primary power of $2 \mathrm{~J}$, uses $50 \mathrm{~nJ}$ to send or receive one bit. The energy consumption of amplifier $\quad \xi$ a m p $\quad=0.0013 \mathrm{pJ} / \mathrm{bit} / \mathrm{m}^{2}$, $\xi f s=10 \mathrm{pJ} / \mathrm{bit} / \mathrm{m}^{2}$ for the purpose of sending the data farther enough. The size of each packet is 500 bytes. The size of packet head is 25 bytes. All nodes are once placed, they can't move. The node dies when its remaining power is equal to $1 \%$ of initial power. The simulation compares the life cycle of these two kinds of algorithms to send equal amount of information under the condition of equal initial power, and the power consumption to send equal amount of information. The result of the simulation is shown as Fig.3.

The result of LAC-T and LAC protocol after 100 tests is shown as Fig.2. According to the power consumption per round, we know that the amount of power consumption of LAC-T algorithm is obviously less than LAC algorithm, thus it can be seen that LAC-T could effectively balance node power consumption distribution, reduce the network's overall energy consumption, extend network lifetime, more suitable for the detection of intrusion, firewall monitoring system. So the LAC-T is more effectively and useful.

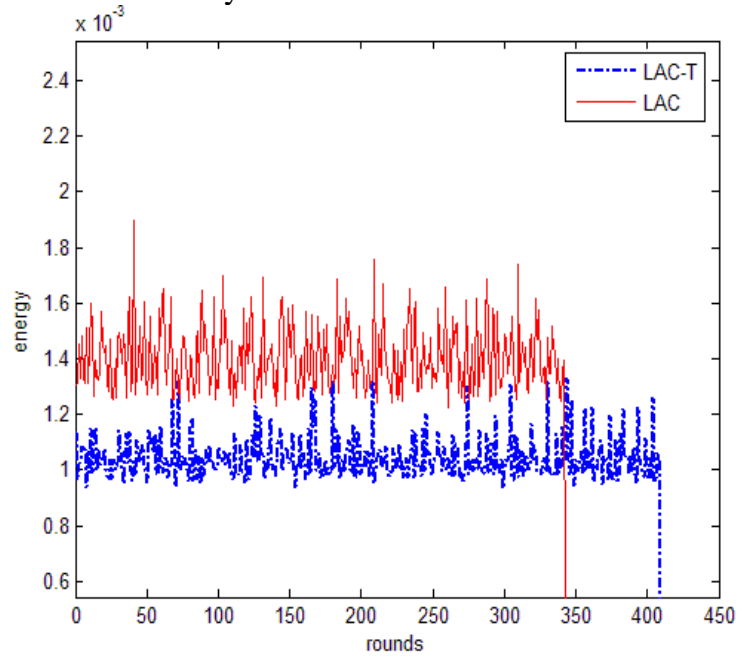

Fig.2. Power consumption per round to send equal amount of information. 


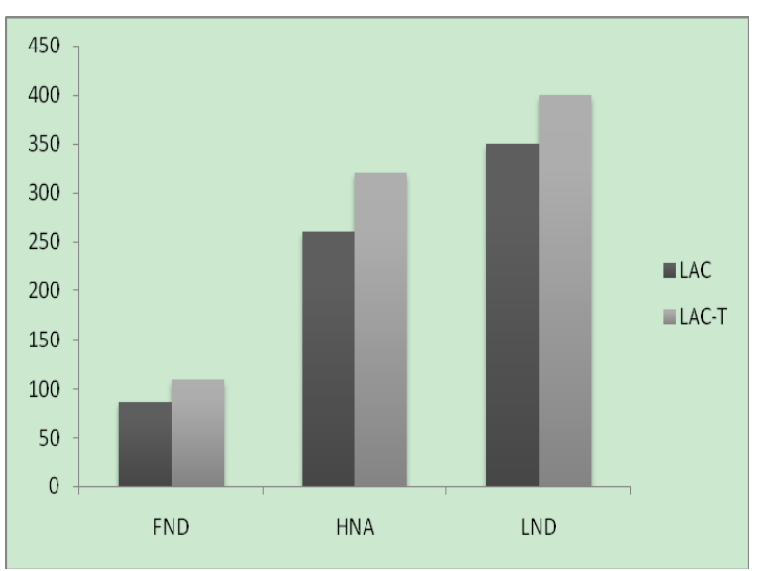

Fig.3. The life cycle of each algorithm with equal initial power.

From Fig. 3 we can see that the first node dies in $85^{\text {th }}$ round of LAC algorithm, and in $110^{\text {th }}$ round of LAC-T algorithm. The life time of first node is increased by approximately 30\%. There left half nodes after 260 rounds of LAC, and after 320 rounds of LAC-T, increasing by approximately $23 \%$. The death time of last node is in $350^{\text {th }}$ round of LAC algorithm, and in $400^{\text {th }}$ round of LAC-T algorithm, increasing by approximately $13 \%$. It reflects from above that LEACH-D's node power consumption is more even than LEACH's, and life cycle of the former is longer than the latter's.

\section{CONCLUSION}

In this article, we present an algorithm of hierarchical topology structure (LAC-T) which based on the nodes distribution in the wireless sensor network. In this algorithm, the cluster head election and cluster radius confirmed are based on nodes distribution, and the approach of communication of cluster heads is multi-hop routing protocol. From the result of the stimulation, we can see that the LAC-T algorithm could effectively reduce the whole power consumption of WSNs, especially suitable for small size WSNs. But in large size WSNs, we need to set other parameters to meet the need of practical communication network [9], [10]. We need to do further research on cluster heads generative mechanism, quantity optimization control, and designing message structure in the practical communication process.

\section{REFERENCES}

[1] Wendi B. Heinzelman. An application-specific protocol architectures for wireless: networks [D]. Boston: Massachusetts institute of Technology, 2002.

[2] Handy J, Haase M, Timmermann D. Low energy adaptive clustering hierarchy with deterministic cluster head selection [C]//Proc of the 4 the International Workshop on Mobile and Wireless Communications of Network. 2002: 368-372.

[3] YOUNG IO, FAHMY S. HEED: a hybrid, energy-efficient distributed clustering approach for Ad hoc sensor networks [J]. IEEE Trans on Mobile Computing, 2004, 3 (4): 366-379.

[4] C. Wang, K.S sohraby and B. Li etc, "A survey of transport protocols for wireless sensor networks," IEEE Network [J], vol.20, pp.34-40, 2006.

[5] C.Y. Wan, A. T. Campbell, and L.Krishnamurthy," PSFQ: A reliable transport protocol for wireless sensor networks[C],"Proc.ACM Intern. Workshop on Wireless. Sensor Network. Appl., pp.1-11, 2002.

[6] F. Stann and J. Heidemann," RMST: Reliable Data Transport in Sensor Networks[C],"1st IEEE International Workshop on Sensor Net Protocols and Applications, 2003.

[7] Y. Sankarasubramaniam, O. B. Akan, and I. F. Akyildiz, " ESRT: Event-to-sink reliable transport in wireless sensor networks[C]," Proc. Int. Symp. Mobile Ad Hoc Networking Computer, pp.177-188, 2003.

[8] C. T. Ee and R. Bajcsy, "Congestion control and fairness for many-to-one routing in sensor networks[C]," in Proceedings of the 2nd international conference on Embedded networked sensor systems Baltimore, MD, USA: ACM, 2004.

[9] Wenwen Dai, Kaihua Xu, James J. (Jong Hyuk) Park, Naixue Xiong and Yuhua Liu. "A Ring and Clustering Routing Scheme based on Data Field in Sensor Network." The 3rd FTRA International Conference on Computer Science and its Applications (CSA-11), Dec. 2011, vol. 114, Part 1.

[10] Yuhua liu, Zhenrong luo, Kaihua xu and Lilong chen, "A Reliable Clustering Algorithm base on LEACH Protocolin Wireless Mobile Sensor Networks," The 2010 International Conference on Mechanical and Electrical Technology(ICMET 2010),IEEE Computer Society Singapore, September 10-12, 2010 